\title{
A comparison of solar proxy-magnetometry diagnostics
}

\author{
J. Leenaarts ${ }^{1}$, R. J. Rutten ${ }^{1,2}$, M. Carlsson ${ }^{2,3}$, and H. Uitenbroek ${ }^{4}$ \\ 1 Sterrekundig Instituut, Postbus 80 000, 3508 TA Utrecht, The Netherlands \\ e-mail: J.Leenaarts@astro.uu.nl \\ 2 Institute of Theoretical Astrophysics, University of Oslo, PO Box 1029, Blindern, 0315 Oslo, Norway \\ 3 Center of Mathematics for Applications, University of Oslo, PO Box 1053, Blindern, 0316 Oslo, Norway \\ 4 NSO/Sacramento Peak, PO Box 62, Sunspot, NM 88349-0062, USA
}

Received 10 March 2006 / Accepted 26 April 2006

\section{ABSTRACT}

\begin{abstract}
Aims. We test various proxy-magnetometry diagnostics, i.e., brightness signatures of small-scale magnetic elements, for studying magnetic field structures in the solar photosphere.

Methods. Images are numerically synthesized from a 3D solar magneto-convection simulation for, respectively, the $\mathrm{G}$ band at $430.5 \mathrm{~nm}$, the $\mathrm{CN}$ band at $388.3 \mathrm{~nm}$, and the blue wings of the $\mathrm{H} \alpha, \mathrm{H} \beta$, Ca II $\mathrm{H}$, and Ca II $854.2 \mathrm{~nm}$ lines.

Results. Both visual comparison and scatter diagrams of the computed intensity versus the magnetic field strength show that, in particular for somewhat spatially extended magnetic elements, the blue $\mathrm{H} \alpha$ wing presents the best proxy-magnetometry diagnostic, followed by the blue wing of $\mathrm{H} \beta$. The latter yields higher diffraction-limit resolution.

Conclusions. We recommend using the blue $\mathrm{H} \alpha$ wing to locate and track small-scale photospheric magnetic elements through their brightness appearance.
\end{abstract}

Key words. Sun: magnetic fields - Sun: granulation - Sun: photosphere

\section{Introduction}

Small bright features that appear within intergranular lanes on solar photospheric filtergrams taken in the $\mathrm{G}$ band of $\mathrm{CH}$ lines at $430.5 \mathrm{~nm}$ are habitually used as proxy diagnostics to study smallscale magnetic structure (e.g., Muller \& Roudier 1984; Berger \& Title 2001; Berger et al. 2004). Such "G-band bright points" have been faithfully reproduced through spectral synthesis applied to 3D magneto-convection simulations of the solar photosphere, at disk center by Schüssler et al. (2003) and Shelyag et al. (2004), and also towards the solar limb by Carlsson et al. (2004). Photospheric magnetic elements appear as bright points also in the wings of strong lines including $\mathrm{Ca}$ II $\mathrm{H} \& \mathrm{~K}$, the $\mathrm{Ca}$ II infrared triplet, the $\mathrm{Mg} \mathrm{I} b$ triplet, and the hydrogen Balmer lines. The CN band at $388.3 \mathrm{~nm}$ shows them also (Rutten et al. 2001), according to Zakharov et al. (2005) even at appreciably higher contrast than the G band but Uitenbroek \& Tritschler (2006) attribute this difference instead to the erroneous inclusion of the $\mathrm{H} \zeta$ line in the filter passband of Zakharov et al. (2005).

In Leenaarts et al. (2006, henceforth Paper I) we compared bright-point visibility in the $\mathrm{G}$ band and the blue wing of $\mathrm{H} \alpha$ using Dutch Open Telescope images. We found that bright points may appear prominently in the $\mathrm{H} \alpha$ wing even when their G-band counterparts are only intermittently present or fully absent. Synthetic $\mathrm{H} \alpha$ images computed from a magneto-convection simulation showed good qualitative agreement with these observations, although not fully matching the observed $\mathrm{H} \alpha$ bright-point contrast. Further analysis demonstrated how magnetic-element brightness enhancement, low granulation contrast, and absence of granular brightness reversal combine to make magnetic elements stand out clearly in this diagnostic.

The $\mathrm{H} \beta$ line has similar formation characteristics as $\mathrm{H} \alpha$ while its shorter wavelength (486.1 nm versus $656.3 \mathrm{~nm}$ for $\mathrm{H} \alpha$ ) yields better angular resolution at the telescopic diffraction limit.
This motivates the test of $\mathrm{H} \beta$ as proxy-magnetometer reported here, performed through image synthesis from a numerical simulation. We extend this appraisal of bright-point response to the wings of $\mathrm{Ca}$ II $\mathrm{H}$ and $\mathrm{Ca}$ II $854.2 \mathrm{~nm}$, selecting the wavelengths shown in Fig. 1. The extended Ca II H \& $\mathrm{K}$ wings merit testing since they provide well-spaced sampling throughout the photosphere (Sheminova et al. 2005). Ca II 854.2 shows $\mathrm{H} \alpha$-like mottles at line center and bright points in its wings, for example in IBIS narrow-band imagery ${ }^{1}$, making direct comparison with $\mathrm{H} \alpha$ of interest. We add $\mathrm{H} \alpha$, G-band and $\mathrm{CN}$-band image synthesis for comparison.

\section{Methods}

We use the same snapshot from a three-dimensional magnetohydrodynamical convection simulation by Stein \& Nordlund (1998) used by also by Carlsson et al. (2004), Uitenbroek \& Tritschler (2006), and in Paper I. The simulation included LTE ionization and non-grey LTE radiative transfer. It was started with a homogeneous vertical magnetic field of $250 \mathrm{G}$. The snapshot used here resulted after considerable evolution in which the field was swept into intergranular concentrations. The simulation snapshot has an horizontal extent of $6 \times 6 \mathrm{Mm}$ and ranges in height from $2.5 \mathrm{Mm}$ below to $0.5 \mathrm{Mm}$ above mean optical depth $\left\langle\tau_{500}\right\rangle=1$. Figure 2 shows the magnitude of the magnetic field at the local $\tau_{500}=1$ surface. The field has ribbon-shaped kilo-Gauss concentrations along edges of mesogranules and strengths of order $30 \mathrm{G}$ within granular interiors. The right-hand panel results from applying a binary mask passing all pixels with $|B|>1 \mathrm{kG}$ (white). We call these locations magnetic elements, and refer to the mask complement (black) as granulation.

\footnotetext{
1 http://wWw .arcetri astro.it/science/solare/IBIS/ ibis.gallery.html
} 

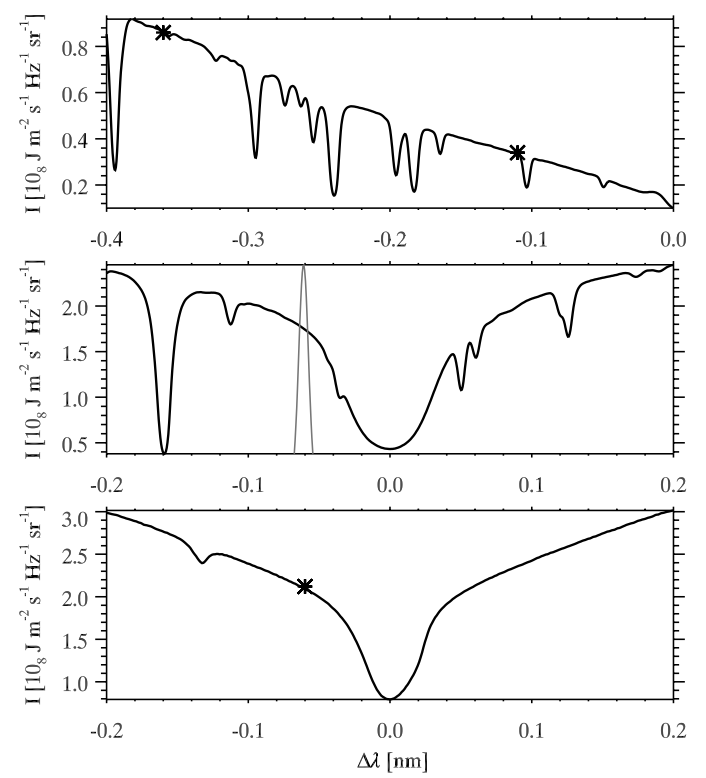

Fig. 1. Spatially averaged disk-center intensity profiles from the NSO Fourier Transform Spectrometer atlas calibrated by Neckel \& Labs (1984). Upper panel: blue wing of Ca II H. The two asterisks mark the wavelengths used for image synthesis. Middle panel: $\mathrm{H} \beta$, with the transmission function used for image synthesis. Lower panel: Ca II 854.2. The asterisk marks the synthesis wavelength.
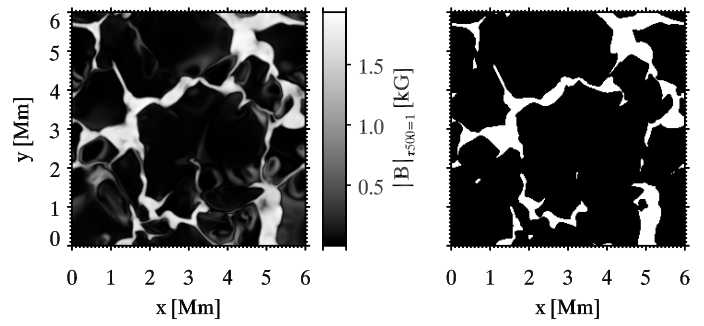

Fig. 2. Left: map of the absolute field strength at $\tau_{500}=1$. Right: mask showing all locations in the 3D simulation with absolute magnetic field strength at $\tau_{500}=1$ exceeding $1 \mathrm{kG}$.

We perform 3D LTE radiative transfer calculations on this simulation cube using the RH code of Uitenbroek (2001) to synthesize disk-center intensity images in the inner and outer blue wing of $\mathrm{CaII} \mathrm{H}$ (at $\Delta \lambda=-0.11 \mathrm{~nm}$ and $-0.36 \mathrm{~nm}$ ), the blue wing of Ca II $854.2(\Delta \lambda=-0.09 \mathrm{~nm})$ and the blue wing of $\mathrm{H} \beta$ $(\Delta \lambda=-0.06 \mathrm{~nm})$. The Ca II $\mathrm{H}$ and $\mathrm{Ca}$ II 854.2 images were computed for a single wavelength, the $\mathrm{H} \beta$ image was synthesized by spectral integration over a Gaussian passband of $0.009 \mathrm{~nm}$ $F W H M$. Figure 1 shows these spectral selections. They are free of blends.

The validity of LTE was confirmed for the $\mathrm{H} \beta$ wing through detailed 2D NLTE computation as in Paper I. The validity of LTE for the Ca II lines has not been tested, but in the FAL-C model of Fontenla et al. (1993) both lines form very close to LTE at the wing wavelengths used here, suggesting that LTE is also a good approximation for our simulation cube.

We also employ synthetic CN-band and G-band images computed by Uitenbroek \& Tritschler (2006) from the same simulation cube through spectrally integrating the emergent intensity over $1 \mathrm{~nm} F W H M$ generalized Lorentzian passbands centered at the respective bandheads, and a synthetic $\mathrm{H} \alpha$-wing image from Paper I for a $0.025 \mathrm{~nm} F W H M$ Gaussian passband centered at $\Delta \lambda=-0.08 \mathrm{~nm}$ from line center. These different diagnostics all form approximately $100 \mathrm{~km}$ higher than the local continuum (Uitenbroek \& Tritschler 2006; Leenaarts et al. 2006), except for the inner $\mathrm{Ca}$ II $\mathrm{H}$ wing which originates about $250 \mathrm{~km}$ above $\left\langle\tau_{500}\right\rangle=1$ (Leenaarts \& Wedemeyer-Böhm 2005).

\section{Results}

Figure 3 shows the computed synthetic intensity images ordered in wavelength from top to bottom. The columns contain different image displays: without spatial degradation (left), convoluted with an Airy function corresponding to a $1 \mathrm{~m}$ telescope aperture (center), and difference images (right). The latter are obtained as $I_{\text {diff }}=I /\langle I\rangle-C I_{\text {cont }} /\left\langle I_{\text {cont }}\right\rangle$ by subtracting images synthesized with $1-\mathrm{m}$ smearing at a nearby continuum wavelength from the smeared image in the center column applying empirically determined factors $C$ following e.g., Berger et al. (1998). The continua used in these subtractions are at $388.3 \mathrm{~nm}$ for the $\mathrm{CN}$ band $(C=0.7)$ and the outer Ca II H wing $(C=0.5), 430.5 \mathrm{~nm}$ for the $\mathrm{G}$ band $(C=0.65), 500 \mathrm{~nm}$ for $\mathrm{H} \beta(C=0.35)$, and $654 \mathrm{~nm}$ for $\mathrm{H} \alpha(C=0.2)$ and $\mathrm{Ca}$ II $854.2(C=0.4)$. Figure 3 has no difference panel for the inner $\mathrm{Ca}$ II $\mathrm{H}$ wing because it shows reversed instead of normal granulation so that subtraction does not help. The upper number in the upper-left corner of each panel is the relative rms intensity variation $\sqrt{\sum_{n}(I-\langle I\rangle)^{2} /\langle I\rangle^{2} / n}$ of the granulation alone. The lower number specifies the average contrast of the bright points over the granulation defined as $\left\langle I_{\mathrm{BP}}\right\rangle /\left\langle I_{\mathrm{GR}}\right\rangle-1$.

Visual inspection of the images and comparison with Fig. 2 shows that the $\mathrm{H} \alpha$ wing is the best diagnostic to identify magnetic elements, in particular less compact ones. Its low granular intensity modulation makes the bright points stand out even though their contrast over the granulation is rather small. The next best is the $\mathrm{H} \beta$ wing. In the $\mathrm{G}$ band and the $\mathrm{CN}$ band the more extended field concentrations are not brighter than the granulation, for example at $(x, y)=(5,0.5)$ and $(4.5,5.5)$ (cf. Fig. 8 and the discussion thereof in Berger et al. 2004). The outer $\mathrm{Ca}$ II $\mathrm{H}$ wing shows a scene somewhat between the molecular bands and $\mathrm{H} \beta$, with compact magnetic elements appearing bright and the more extended elements only slightly brighter than granules. The bright points in the inner $\mathrm{Ca}$ II $\mathrm{H}$ wing are more diffuse and have different morphology than in the other diagnostics.

All diagnostics perform better in the difference images in the third column of Fig. 3, but also in these the $\mathrm{G}$ band, $\mathrm{CN}$ band and outer $\mathrm{Ca}$ II $\mathrm{H}$ wing perform worse than the Balmer wings. In $\mathrm{H} \beta$ the magnetic areas appear almost the same as in $\mathrm{H} \alpha$ but sharper, whereas the Ca II 854.2 wing suffers from lower resolution than $\mathrm{H} \alpha$. Note that all difference images show a Y-shaped brightness structure centered at $(x, y)=(1,2)$ which results at least partially not from magnetism but from the combination of a dark granular lane in the continua and reversed-granulation brightening at larger height.

Figure 4 shows scatter diagrams of normalized intensity against magnetic field strength per smeared-image pixel. The first column shows these for the images in the center column of Fig. 3. All diagnostics show a large cluster of pixels at nearzero field strength, caused by the granulation. There is a second cluster at high field strengths, caused by the magnetic elements. The higher the intensity of the second cluster is raised above the granulation, the better is the corresponding diagnostic as a proxy magnetometer. This means in terms of intensity distribution functions (DF) that the more the low $B$ DF (blue) is separated from the high $B$ DF (red), the better is the corresponding 

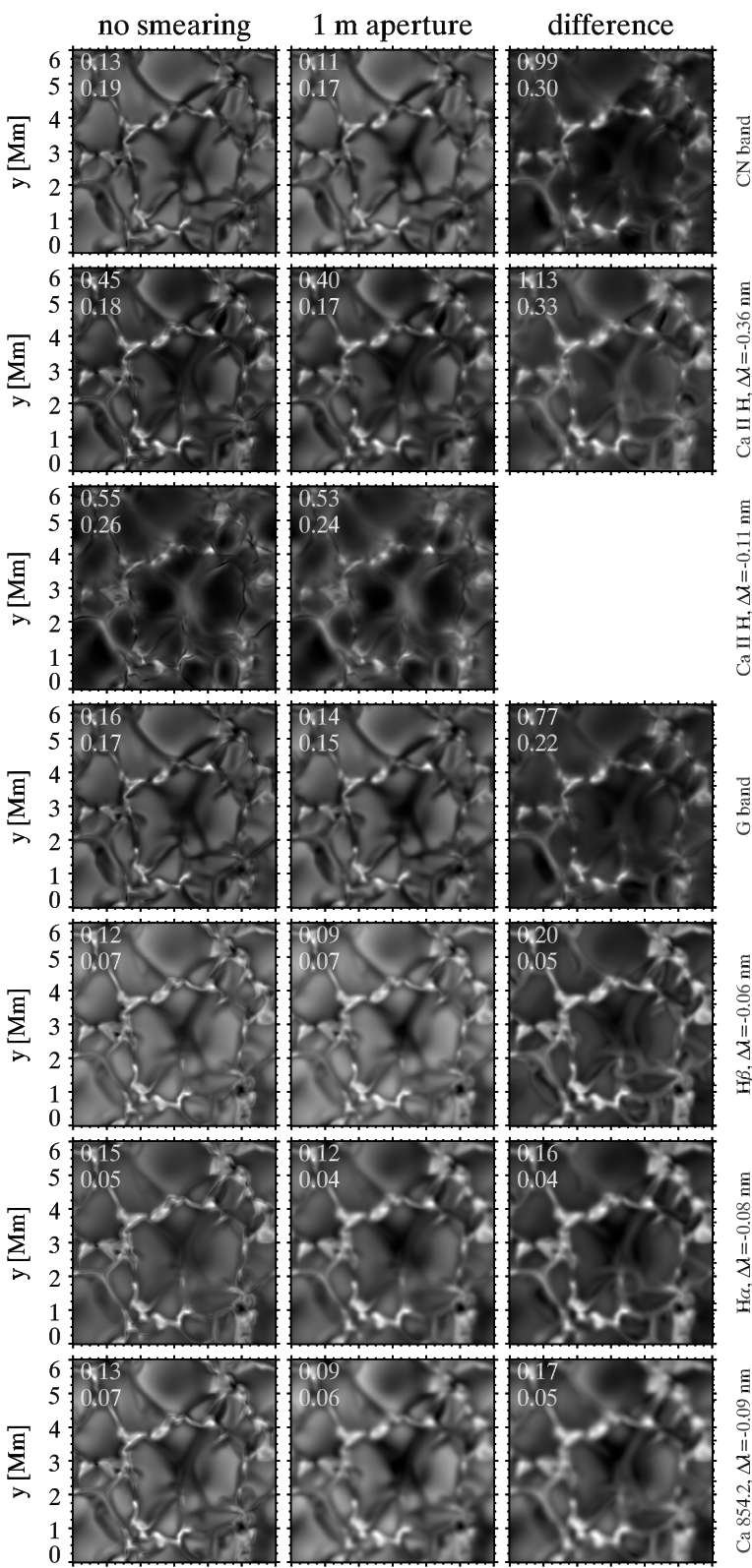

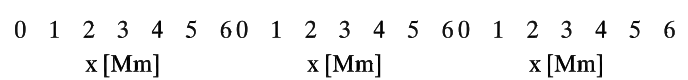

Fig. 3. Synthetic images for different spectral diagnostics identified per row at the right. The $\mathrm{CN}$-band and G-band images are taken from Uitenbroek \& Tritschler (2006). All images are grey-scaled to maximum display contrast. The rms intensity variation of the granulation (upper number) and the mean intensity excess of the bright points over the granulation (lower number) is indicated in each upper-left corner. Left: no spatial smearing. Middle: smeared with an Airy function corresponding to $1-\mathrm{m}$ aperture. Right: image constructed by subtracting a scaled synthetic continuum image from the one in the center column.

diagnostic as a proxy-magnetometer. $\mathrm{H} \alpha$ performs best in this regard. The diagrams in the second column show that subtracting continuum images greatly improves the bright-point visibility, raising the strong-field-pixel cluster above the granulation also for the outer $\mathrm{Ca}$ II $\mathrm{H}, \mathrm{H} \beta$, and Ca II 854.2 wings.

\section{Discussion and conclusion}

We conclude that the blue wing of $\mathrm{H} \alpha$ provides the best magnetic-element visibility. Second best is the blue wing of
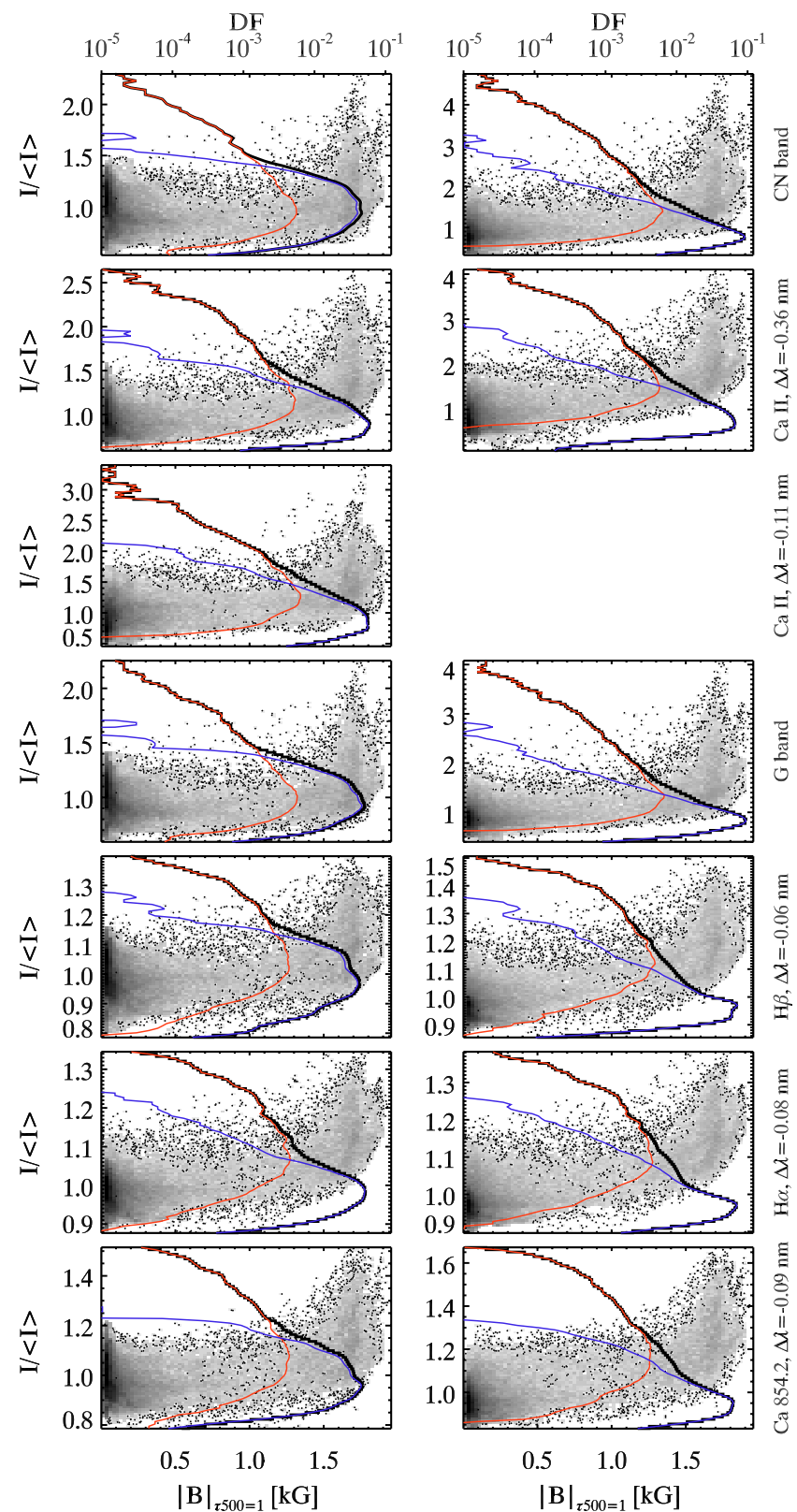

Fig. 4. Scatter diagrams of normalized intensity $I /\langle I\rangle$ versus magnetic field strength at $\tau_{500}=1$ per pixel. To avoid plot overcrowding an inverted logarithmic grayscale is used, i.e., black areas have the highest point density. Left: intensity for $1 \mathrm{~m}$ aperture. Right: difference image. The curves indicate the normalized intensity distribution function for the corresponding image on a logarithmic scale shown at the top. Black: all pixels. Blue: pixels with $|B|_{\tau 500=1}<1 \mathrm{kG}$. Red: with $|B|_{\tau 500=1}>1 \mathrm{kG}$.

$\mathrm{H} \beta$ which offers, especially in the difference image, similar bright-point visibility at higher resolution. The other diagnostics suffer from lower resolution (Ca II 854.2), contamination with reversed granulation (inner $\mathrm{Ca}$ II $\mathrm{H}$ wing), or lack of enhancement in extended field concentrations ( $\mathrm{G}$ band, $\mathrm{CN}$ band, outer Ca II H wing).

The reasons why the blue $\mathrm{H} \alpha$ wing performs so well were analyzed in detail in Paper I. They are a combination of low Planck function sensitivity to temperature, steep-wing Doppler compensation of granular temperature contrast, a deep opacity gap in the upper photosphere due to high excitation energy, small brightness response to temperature increase through 


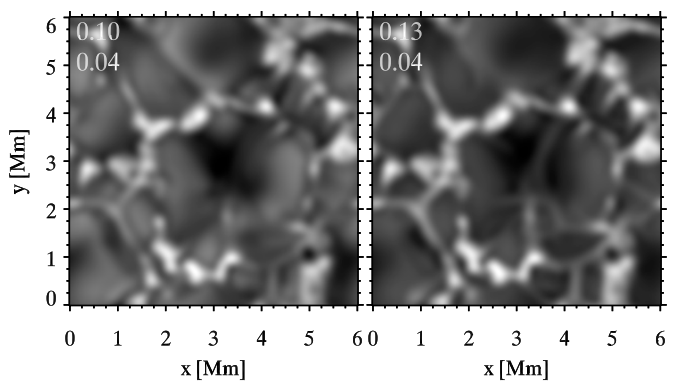

Fig. 5. Intensity and difference images for the $\mathrm{H} \alpha$ wing, convoluted with an Airy function corresponding to the Solar-B aperture of $50 \mathrm{~cm}$. Left: $\mathrm{H} \alpha$ wing intensity. Right: $\mathrm{H} \alpha$ wing minus continuum. The rms intensity variation of the granulation (upper number) and the mean intensity excess of the bright points over the granulation (lower number) is indicated in each upper-left corner.

concomitant opacity increase, and reduction of collisional broadening within magnetic elements. These factors combine to brighten magnetic elements against a very "flat" non-reversed granular background in $\mathrm{H} \alpha$. The same effects (except the first) apply also to $\mathrm{H} \beta$.

In hindsight, the absence of reversed granulation in $\mathrm{H} \beta$ was already described by Evans \& Catalano (1972) as absence of "oddities" affecting the Ca II H\& K and Mg I b wings. They regarded $\mathrm{H} \beta$ as non-peculiar, but in fact reversed granulation results naturally from convection into a radiative overshoot layer (Leenaarts \& Wedemeyer-Böhm 2005); its absence in the Balmer lines is peculiar. At the time, Thomas (1972) offered a complex NLTE interpretation in an accompanying note, but the actual explanation (given in Paper I) is simple and does not involve NLTE.

The Balmer line wings have the drawback of requiring narrower spectral passband than the molecular bands. They are also more susceptible to showing overlying chromospheric mottles than the molecular bands and the wings of $\mathrm{H} \& \mathrm{~K}$. Nevertheless, the blue $\mathrm{H} \alpha$ wing turns out the best proxy-magnetometer to locate and track spatially extended magnetic elements. We so champion this "chromospheric" line as photospheric diagnostic. Figure 5 shows synthetic $\mathrm{H} \alpha$ images at the resolution of the $50-\mathrm{cm}$ Solar-B telescope. It will be of interest to compare Solar-B tunable-filter imaging in the wing of $\mathrm{H} \alpha$ with Solar-B scanning magnetometry. The first may complement the second as high-cadence full-field diagnostic.

\section{References}

Berger, T. E., \& Title, A. M. 2001, ApJ, 553, 449

Berger, T. E., Löfdahl, M. G., Shine, R. A., \& Title, A. M. 1998, ApJ, 506, 439 Berger, T. E., Rouppe van der Voort, L. H. M., Löfdahl, M. G., et al. 2004, A\&A, 428,613

Carlsson, M., Stein, R. F., Nordlund, Å., \& Scharmer, G. B. 2004, ApJ, 610, L137

Evans, J. W., \& Catalano, C. P. 1972, Sol. Phys., 27, 299

Fontenla, J. M., Avrett, E. H., \& Loeser, R. 1993, ApJ, 406, 319

Leenaarts, J., \& Wedemeyer-Böhm, S. 2005, A\&A, 431, 687

Leenaarts, J., Rutten, R. J., Sütterlin, P., Carlsson, M., \& Uitenbroek, H. 2006, A\&A, 449, 1209 (Paper I)

Muller, R., \& Roudier, T. 1984, Sol. Phys., 94, 33

Neckel, H., \& Labs, D. 1984, Sol. Phys., 90, 205

Rutten, R. J., Kiselman, D., Rouppe van der Voort, L., \& Plez, B. 2001, in Advanced Solar Polarimetry - Theory, Observation, and Instrumentation, ASP Conf. Ser., 236, 445

Schüssler, M., Shelyag, S., Berdyugina, S., Vögler, A., \& Solanki, S. K. 2003, ApJ, 597, L173

Shelyag, S., Schüssler, M., Solanki, S. K., Berdyugina, S. V., \& Vögler, A. 2004, A\&A, 427, 335

Sheminova, V. A., Rutten, R. J., \& Rouppe van der Voort, L. H. M. 2005, A\&A, 437, 1069

Stein, R. F., \& Nordlund, A. 1998, ApJ, 499, 914

Thomas, R. N. 1972, Sol. Phys., 27, 303

Uitenbroek, H. 2001, ApJ, 557, 389

Uitenbroek, H., \& Tritschler, A. 2006, ApJ, 639, 525

Zakharov, V., Gandorfer, A., Solanki, S. K., \& Löfdahl, M. 2005, A\&A, 437, L43 